\title{
Crystal structure of 3-diphenylamino-benzo[de]anthracen-7-one, $\mathrm{C}_{29} \mathrm{H}_{19} \mathrm{NO}$
}

\author{
M.-X. Yu* \\ Zhejiang University, Department of Chemistry, Hangzhou, Zhejiang, 310027 P. R. China
}

Received January 9, 2004, accepted and available on-line February 11, 2004; CCDC no. 1267/1203
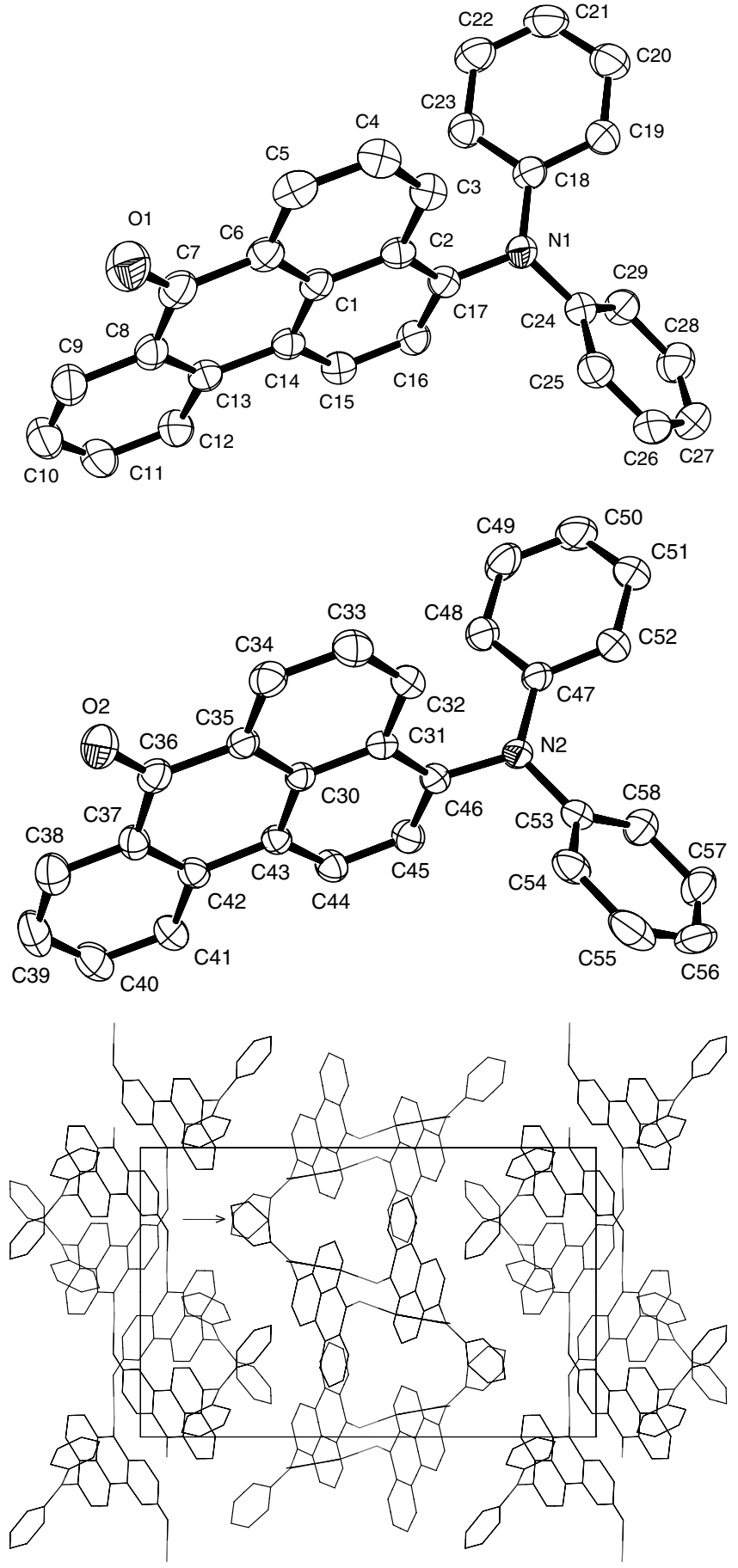

* e-mail: mingxinyu@css.zju.edu.cn

\author{
Abstract \\ $\mathrm{C}_{29} \mathrm{H}_{19} \mathrm{NO}$, monoclinic, $P 12{ }_{1} / c 1$ (no. 14), \\ $a=23.218(4) \AA, b=14.748(2) \AA, c=12.094(2) \AA$, \\ $\beta=90.986(4)^{\circ}, V=4140.6 \AA^{3}, Z=8$, \\ $R_{\mathrm{gt}}(F)=0.045, w R_{\mathrm{ref}}\left(F^{2}\right)=0.085, T=293 \mathrm{~K}$.
}

\section{Source of material}

To a 25-mL sidearm flask in a dry nitrogen box were added 3bromo-benzo[de]anthracen-7-one $(2.5 \mathrm{mmol})$, diphenyl-amine $(2.50 \mathrm{mmol}), \mathrm{Pd}\left(\mathrm{CH}_{3} \mathrm{COO}\right)_{2}(0.025 \mathrm{mmol}, \mathrm{Pd} / \mathrm{Br}=1 \%)$, tri-tertbutylphosphine $(0.03 \mathrm{mmol})$, and sodium tert-butoxide $(3.0$ mmol). The flask was sealed with a septum and was then moved out of the nitrogen box. $o$-xylene $(10 \mathrm{~mL})$ was injected via a syringe. The reaction mixture was heated and stirred at $120{ }^{\circ} \mathrm{C}$ under nitrogen until the reaction was completed. The reaction mixture was then cooled to room temperature, filtered through a mixture of celite and silica gel pad, and washed with dichloromethane. The filtrate was then washed with water and dried by $\mathrm{MgSO}_{4}$. Concentration of the filtrate on a rotary evaporator followed by washing of the solid material with methanol afforded the desired crude product. The crude product was then dried at $120^{\circ} \mathrm{C}$ in a vacuum and further purified by sublimation. Red crystals were obtained by sublimation at $(4 \ldots 6) \cdot 10^{-3} \mathrm{~Pa}$ for $16 \mathrm{~h}$.

\section{Discussion}

Aromatic amines play a central role in many areas of modern day organic chemistry, including such diverse fields as polymers, photography, medicine and materials [1-3]. Arylamines can be obtained either by the copper-catalyzed Ullmann condensation or palladium-catalyzed cross-coupling reactions [4-9]. Ullmann reactions involve rather high temperatures and prolonged reaction times and are limited to aryl iodides. Palladium-catalyzed $N$ arylation reaction has opened up the possibility of producing a wide variety of arylamines.

In the crystal structure there are two independent molecular units with similar bond lengths and angles (figure, top \& middle).The planes C17/C18/C24 and C46/C47/C53 related to the amine geometry are all perfectly planar, indicating nitrogen atoms have a $s p^{2}$ hybridization. The benzo[de]anthracen-7-one ring systems are co-planar. Dihedral angles among planes of three substituted groups attached atoms of $\mathrm{N} 1$ and $\mathrm{N} 2$ are $60.44^{\circ}, 72.57^{\circ}$, and $83.44^{\circ}$, as well as $68.02^{\circ}, 78.47^{\circ}$ and $82.00^{\circ}$. Therefore such arrangements have little steric crowding. All bond lengths in the compound are in the normal range, though disorder around C55C57 appeared due to the loose packing of the concerning phenyl ring (s. arrow in figure, bottom). In the cell projection along $c$ the connected molecular units I are located along the border and units II in the centre. The two molecular units greatly differ in their packing. The shortest intermolecular contacts thereby are $2.52 \AA$,

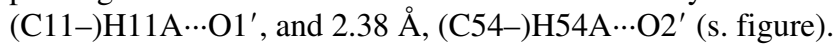


Table 1. Data collection and handling.

Crystal:

Wavelength:

$\mu$ :

Diffractometer, scan mode:

$2 \theta_{\max }$ :

$N(h k l)_{\text {measured }}, N(h k l)_{\text {unique: }}$

Criterion for $I_{\mathrm{obs}}, N(h k l)_{\mathrm{gt}}$ :

$N(\text { param })_{\text {refined: }}$

Programs:

\author{
red columnar, size $0.2 \times 0.3 \times 0.4 \mathrm{~mm}$ \\ Mo $K_{\alpha}$ radiation $(0.71073 \AA)$ \\ $0.77 \mathrm{~cm}^{-1}$ \\ Bruker SMART CCD, $\varphi / \omega$ \\ $50.5^{\circ}$ \\ 18154,6699 \\ $I_{\mathrm{obs}}>2 \sigma\left(I_{\mathrm{obs}}\right), 2724$ \\ 560 \\ SHELX-97 [10], ORTEP-3 [11], \\ WinGX [12]
}

Table 2. Atomic coordinates and displacement parameters (in $\AA^{2}$ ).

\begin{tabular}{llllll}
\hline Atom & Site & $x$ & $y$ & $z$ & $U_{\text {iso }}$ \\
\hline & & & & & \\
$\mathrm{H}(3 \mathrm{~A})$ & $4 e$ & 0.1895 & 0.4957 & 0.2611 & 0.08 \\
$\mathrm{H}(4 \mathrm{~A})$ & $4 e$ & 0.1792 & 0.6306 & 0.3655 & 0.08 \\
$\mathrm{H}(5 \mathrm{~A})$ & $4 e$ & 0.1174 & 0.6311 & 0.5181 & 0.08 \\
$\mathrm{H}(9 \mathrm{~A})$ & $4 e$ & -0.0083 & 0.4774 & 0.7669 & 0.08 \\
$\mathrm{H}(10 \mathrm{~A})$ & $4 e$ & -0.0630 & 0.3466 & 0.8242 & 0.08 \\
$\mathrm{H}(11 \mathrm{~A})$ & $4 e$ & -0.0599 & 0.2129 & 0.7152 & 0.08 \\
$\mathrm{H}(12 \mathrm{~A})$ & $4 e$ & -0.0033 & 0.2106 & 0.5582 & 0.08 \\
$\mathrm{H}(15 \mathrm{~A})$ & $4 e$ & 0.0523 & 0.2083 & 0.4274 & 0.08 \\
$\mathrm{H}(16 \mathrm{~A})$ & $4 e$ & 0.1124 & 0.2061 & 0.2735 & 0.08 \\
$\mathrm{H}(19 \mathrm{~A})$ & $4 e$ & 0.2357 & 0.3779 & 0.0212 & 0.08 \\
$\mathrm{H}(20 \mathrm{~A})$ & $4 e$ & 0.2119 & 0.4691 & -0.1320 & 0.08 \\
$\mathrm{H}(21 \mathrm{~A})$ & $4 e$ & 0.1175 & 0.5318 & -0.1415 & 0.08 \\
$\mathrm{H}(22 \mathrm{~A})$ & $4 e$ & 0.0550 & 0.5092 & -0.0062 & 0.08 \\
$\mathrm{H}(23 \mathrm{~A})$ & $4 e$ & 0.0746 & 0.4166 & 0.1481 & 0.08 \\
$\mathrm{H}(25 \mathrm{~A})$ & $4 e$ & 0.2495 & 0.2846 & 0.3380 & 0.08 \\
$\mathrm{H}(26 \mathrm{~A})$ & $4 e$ & 0.3132 & 0.1617 & 0.3347 & 0.08 \\
$\mathrm{H}(27 \mathrm{~A})$ & $4 e$ & 0.3155 & 0.0645 & 0.1723 & 0.08 \\
$\mathrm{H}(28 \mathrm{~A})$ & $4 e$ & 0.2487 & 0.0919 & 0.0222 & 0.08 \\
$\mathrm{H}(29 \mathrm{~A})$ & $4 e$ & 0.1848 & 0.2162 & 0.0319 & 0.08 \\
& & & & &
\end{tabular}

Table 2. Continued.

\begin{tabular}{llllrl}
\hline Atom & Site & $x$ & $y$ & \multicolumn{1}{l}{$z$} & $U_{\text {iso }}$ \\
\hline & & & & & \\
$\mathrm{H}(32 \mathrm{~A})$ & $4 e$ & 0.3803 & 0.7035 & 0.1262 & 0.08 \\
$\mathrm{H}(33 \mathrm{~A})$ & $4 e$ & 0.4379 & 0.7384 & -0.0233 & 0.08 \\
$\mathrm{H}(34 \mathrm{~A})$ & $4 e$ & 0.4684 & 0.6241 & -0.1477 & 0.08 \\
$\mathrm{H}(38 \mathrm{~A})$ & $4 e$ & 0.4784 & 0.3058 & -0.2536 & 0.08 \\
$\mathrm{H}(39 \mathrm{~A})$ & $4 e$ & 0.4587 & 0.1515 & -0.2141 & 0.08 \\
$\mathrm{H}(40 \mathrm{~A})$ & $4 e$ & 0.4053 & 0.1081 & -0.0538 & 0.08 \\
$\mathrm{H}(41 \mathrm{~A})$ & $4 e$ & 0.3721 & 0.2213 & 0.0642 & 0.08 \\
$\mathrm{H}(44 \mathrm{~A})$ & $4 e$ & 0.3420 & 0.3217 & 0.1604 & 0.08 \\
$\mathrm{H}(45 \mathrm{~A})$ & $4 e$ & 0.3094 & 0.4382 & 0.2722 & 0.08 \\
$\mathrm{H}(48 \mathrm{~A})$ & $4 e$ & 0.2524 & 0.6108 & 0.1102 & 0.08 \\
$\mathrm{H}(49 \mathrm{~A})$ & $4 e$ & 0.1822 & 0.7114 & 0.0730 & 0.08 \\
$\mathrm{H}(50 \mathrm{~A})$ & $4 e$ & 0.1710 & 0.8430 & 0.1877 & 0.08 \\
$\mathrm{H}(51 \mathrm{~A})$ & $4 e$ & 0.2357 & 0.8664 & 0.3304 & 0.08 \\
$\mathrm{H}(52 \mathrm{~A})$ & $4 e$ & 0.3078 & 0.7651 & 0.3670 & 0.08 \\
$\mathrm{H}(54 \mathrm{~A})$ & $4 e$ & 0.4360 & 0.5781 & 0.3219 & 0.08 \\
$\mathrm{H}(55 \mathrm{~A})$ & $4 e$ & 0.4767 & 0.5725 & 0.4814 & 0.08 \\
$\mathrm{H}(56 \mathrm{~A})$ & $4 e$ & 0.4183 & 0.5822 & 0.6513 & 0.08 \\
$\mathrm{H}(57 \mathrm{~A})$ & $4 e$ & 0.3260 & 0.6060 & 0.6396 & 0.08 \\
$\mathrm{H}(58 \mathrm{~A})$ & $4 e$ & 0.2822 & 0.6167 & 0.4611 & 0.08 \\
& & & & &
\end{tabular}

Table 3. Atomic coordinates and displacement parameters (in $\AA^{2}$ ).

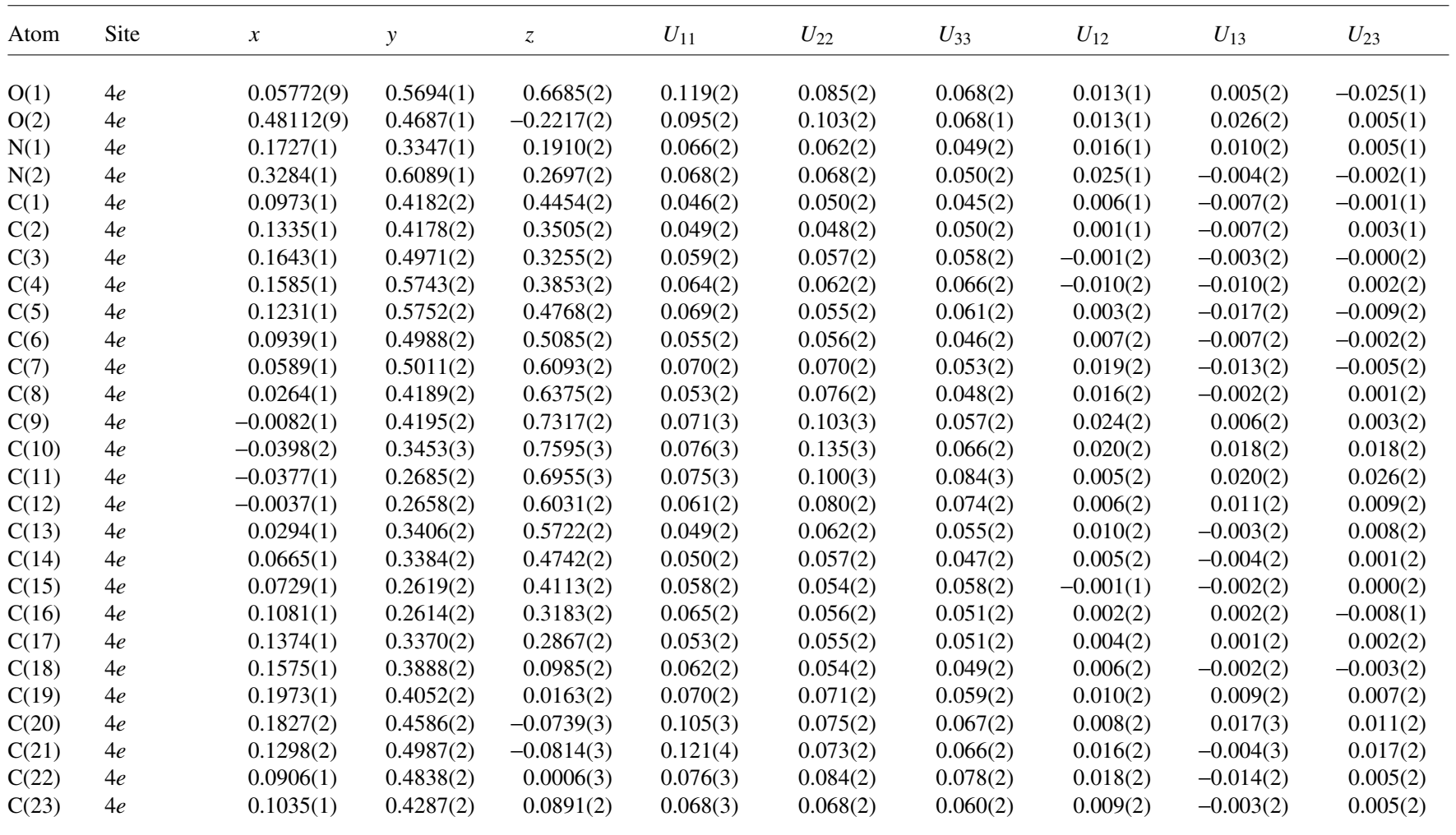


Table 3. Continued.

\begin{tabular}{|c|c|c|c|c|c|c|c|c|c|c|}
\hline Atom & Site & $x$ & $y$ & $z$ & $U_{11}$ & $U_{22}$ & $U_{33}$ & $U_{12}$ & $U_{13}$ & $U_{23}$ \\
\hline $\mathrm{C}(24)$ & $4 e$ & $0.2112(1)$ & $0.2598(2)$ & $0.1837(2)$ & $0.054(2)$ & $0.057(2)$ & $0.052(2)$ & $0.003(2)$ & $0.005(2)$ & $0.001(2)$ \\
\hline$C(25)$ & $4 e$ & $0.2484(1)$ & $0.2427(2)$ & $0.2716(2)$ & $0.059(2)$ & $0.065(2)$ & $0.058(2)$ & $-0.003(2)$ & $-0.002(2)$ & $0.002(2)$ \\
\hline $\mathrm{C}(26)$ & $4 e$ & $0.2857(1)$ & $0.1699(2)$ & $0.2668(3)$ & $0.063(2)$ & $0.090(2)$ & $0.070(2)$ & $0.009(2)$ & $-0.004(2)$ & $0.018(2)$ \\
\hline $\mathrm{C}(27)$ & $4 e$ & $0.2867(2)$ & $0.1149(2)$ & $0.1763(3)$ & $0.082(3)$ & $0.080(2)$ & $0.101(3)$ & $0.031(2)$ & $0.017(3)$ & $0.009(2)$ \\
\hline $\mathrm{C}(28)$ & $4 e$ & $0.2496(2)$ & $0.1312(2)$ & $0.0898(3)$ & $0.096(3)$ & $0.075(2)$ & $0.080(3)$ & $0.019(2)$ & $0.011(3)$ & $-0.013(2)$ \\
\hline $\mathrm{C}(29)$ & $4 e$ & $0.2117(1)$ & $0.2034(2)$ & $0.0918(2)$ & $0.069(2)$ & $0.069(2)$ & $0.060(2)$ & $0.009(2)$ & $-0.005(2)$ & $-0.006(2)$ \\
\hline $\mathrm{C}(31)$ & $4 e$ & $0.3777(1)$ & $0.5652(2)$ & $0.0976(2)$ & $0.052(2)$ & $0.050(2)$ & $0.050(2)$ & $0.005(1)$ & $-0.005(2)$ & $0.005(2)$ \\
\hline $\mathrm{C}(32)$ & $4 e$ & $0.3938(1)$ & $0.6555(2)$ & $0.0758(2)$ & $0.071(2)$ & $0.054(2)$ & $0.064(2)$ & $0.008(2)$ & $0.001(2)$ & $-0.000(2)$ \\
\hline $\mathrm{C}(33)$ & $4 e$ & $0.4265(1)$ & $0.6764(2)$ & $-0.0132(3)$ & $0.087(3)$ & $0.055(2)$ & $0.079(2)$ & $-0.007(2)$ & $0.004(2)$ & $0.008(2)$ \\
\hline $\mathrm{C}(34)$ & $4 e$ & $0.4444(1)$ & $0.6084(2)$ & $-0.0842(2)$ & $0.068(2)$ & $0.069(2)$ & $0.060(2)$ & $-0.001(2)$ & $0.006(2)$ & $0.011(2)$ \\
\hline $\mathrm{C}(35)$ & $4 e$ & $0.4302(1)$ & $0.5192(2)$ & $-0.0658(2)$ & $0.050(2)$ & $0.059(2)$ & $0.048(2)$ & $0.005(1)$ & $-0.001(2)$ & $0.005(2)$ \\
\hline$C(36)$ & $4 e$ & $0.4518(1)$ & $0.4483(2)$ & $-0.1414(2)$ & $0.052(2)$ & $0.080(2)$ & $0.051(2)$ & $0.008(2)$ & $-0.000(2)$ & $0.003(2)$ \\
\hline $\mathrm{C}(37)$ & $4 e$ & $0.4373(1)$ & $0.3533(2)$ & $-0.1168(2)$ & $0.048(2)$ & $0.067(2)$ & $0.057(2)$ & $0.006(2)$ & $-0.004(2)$ & $-0.011(2)$ \\
\hline $\mathrm{C}(38)$ & $4 e$ & $0.4568(1)$ & $0.2847(2)$ & $-0.1871(2)$ & $0.070(3)$ & $0.088(2)$ & $0.075(2)$ & $0.003(2)$ & $-0.003(2)$ & $-0.019(2)$ \\
\hline $\mathrm{C}(40)$ & $4 e$ & $0.4139(2)$ & $0.1727(2)$ & $-0.0739(3)$ & $0.094(3)$ & $0.058(2)$ & $0.109(3)$ & $0.005(2)$ & $-0.006(2)$ & $-0.017(2)$ \\
\hline $\mathrm{C}(41)$ & $4 e$ & $0.3941(1)$ & $0.2388(2)$ & $-0.0034(2)$ & $0.080(3)$ & $0.056(2)$ & $0.081(2)$ & $0.005(2)$ & $0.000(2)$ & $-0.007(2)$ \\
\hline $\mathrm{C}(42)$ & $4 e$ & $0.4053(1)$ & $0.3310(2)$ & $-0.0240(2)$ & $0.050(2)$ & $0.054(2)$ & $0.060(2)$ & $0.006(2)$ & $-0.009(2)$ & $-0.005(2)$ \\
\hline $\mathrm{C}(43)$ & $4 e$ & $0.3836(1)$ & $0.4029(2)$ & $0.0492(2)$ & $0.045(2)$ & $0.050(2)$ & $0.052(2)$ & $0.003(1)$ & $-0.004(2)$ & $-0.001(2)$ \\
\hline $\mathrm{C}(44)$ & $4 e$ & $0.3510(1)$ & $0.3839(2)$ & $0.1396(2)$ & $0.068(2)$ & $0.050(2)$ & $0.066(2)$ & $-0.001(2)$ & $0.010(2)$ & $0.004(2)$ \\
\hline $\mathrm{C}(45)$ & $4 e$ & $0.3322(1)$ & $0.4524(2)$ & $0.2102(2)$ & $0.067(2)$ & $0.066(2)$ & $0.062(2)$ & $0.006(2)$ & $0.015(2)$ & $0.005(2)$ \\
\hline $\mathrm{C}(46)$ & $4 e$ & $0.3448(1)$ & $0.5414(2)$ & $0.1912(2)$ & $0.060(2)$ & $0.056(2)$ & $0.052(2)$ & $0.011(2)$ & $0.003(2)$ & $0.001(2)$ \\
\hline $\mathrm{C}(47)$ & $4 e$ & $0.2858(1)$ & $0.6739(2)$ & $0.2443(3)$ & $0.060(3)$ & $0.066(2)$ & $0.048(2)$ & $0.015(2)$ & $-0.002(2)$ & $0.003(2)$ \\
\hline $\mathrm{C}(48)$ & $4 e$ & $0.2481(2)$ & $0.6604(2)$ & $0.1565(3)$ & $0.077(3)$ & $0.083(2)$ & $0.062(2)$ & $0.020(2)$ & $0.007(2)$ & $0.002(2)$ \\
\hline $\mathrm{C}(49)$ & $4 e$ & $0.2052(2)$ & $0.7244(3)$ & $0.1340(3)$ & $0.085(3)$ & $0.134(3)$ & $0.062(2)$ & $0.030(2)$ & $-0.018(2)$ & $-0.001(2)$ \\
\hline $\mathrm{C}(50)$ & $4 e$ & $0.1993(2)$ & $0.7987(3)$ & $0.1988(4)$ & $0.113(4)$ & $0.122(3)$ & $0.087(3)$ & $0.063(3)$ & $-0.001(3)$ & $0.013(3)$ \\
\hline $\mathrm{C}(51)$ & $4 e$ & $0.2371(2)$ & $0.8129(2)$ & $0.2843(3)$ & $0.120(4)$ & $0.081(3)$ & $0.084(3)$ & $0.043(2)$ & $-0.007(3)$ & $-0.003(2)$ \\
\hline$C(52)$ & $4 e$ & $0.2805(1)$ & $0.7516(2)$ & $0.3073(2)$ & $0.086(3)$ & $0.068(2)$ & $0.069(2)$ & $0.020(2)$ & $-0.002(2)$ & $-0.005(2)$ \\
\hline $\mathrm{C}(53)$ & $4 e$ & $0.3532(1)$ & $0.6011(2)$ & $0.3770(3)$ & $0.055(2)$ & $0.046(2)$ & $0.064(2)$ & $0.007(2)$ & $-0.002(2)$ & $0.001(2)$ \\
\hline $\mathrm{C}(54)$ & $4 e$ & $0.4113(2)$ & $0.5849(2)$ & $0.3878(3)$ & $0.059(3)$ & $0.056(2)$ & $0.099(3)$ & $0.009(2)$ & $-0.024(3)$ & $-0.013(2)$ \\
\hline $\mathrm{C}(55)$ & $4 e$ & $0.4362(2)$ & $0.5767(2)$ & $0.4918(5)$ & $0.085(3)$ & $0.062(2)$ & $0.175(5)$ & $0.021(2)$ & $-0.049(4)$ & $-0.032(3)$ \\
\hline $\mathrm{C}(58)$ & $4 e$ & $0.3209(1)$ & $0.6074(2)$ & $0.4725(3)$ & $0.078(3)$ & $0.076(2)$ & $0.063(2)$ & $-0.006(2)$ & $-0.002(2)$ & $0.002(2)$ \\
\hline
\end{tabular}

Acknowledgments. The project is sponsored by the Scientific Research Foundation for the Returned Overseas Chinese Scholars, State Education Ministry and Zhejiang Province. The author thanks Professor Longguan Zhu for X-ray analysis.

\section{References}

1. Wolfe, J. P.; Buchwald, S. L.: Palladium-catalyzed amination of aryl iodides. J. Org. Chem. 61 (1996) 1133-1135.

2. Bellmann, E.; Shaheen, S. E.; Thayumanavan, S.; Barlow, S.; Grubbs, R. H.; Marder, S. R.; Kippelen, B.; Peyghambarian, N.: New triarylaminecontaining polymers as hole transport material in organic light-emitting diodes: effect of polymer structure and cross-linking on device characteristics. Chem. Mater. 10 (1998) 1668-1676.

3. Kim, Y. K.; Shin, D. C.; Kim, S. H.; Ko, C. H.; Yu, H. S.; Chae, Y. S.; Kwon, S. K.: Novel blue emitting material with high color purity. Adv. Mater. 13 (2001) 1690-1693.

4. Paine, A. J.: Mechanisms and models for copper mediated nucleophilic aromatic substitution. 2. Single catalytic species from three different oxidation states of copper in an Ullmann synthesis of triarylamines. J. Am. Chem. Soc. 109 (1987) 1496-1502.

5. Yamamoto, T.; Nishiyama, M.; Koie, Y.: Palladium-catalyzed synthesis of triarylamines from aryl halides and diarylamines. Tetrahedron Lett. 39 (1998) 2367-2370.

6. Thomas, K. R. J.; Lin, J. T.; Tao, Y. T.; Ko, C. K.: Light-emitting carbazol derivatives: Potential electroluminescent materials. J. Am. Chem. Soc. 123 (2001) 9409-9411.
7. Wolfe, J. P.; Wagaw, S.; Marcoux, J. F.; Buchwald, S. L.: Rational development of practical catalysts for aromatic carbon-nitrogen bond formation. Acc. Chem. Res. 31 (1998) 865-818.

8. Hartwig, J. F.: Transition metal catalyzed synthesis of arylamine and aryl ethers from aryl halides and triflates: Scope and mechanism. Angew. Chem., Int. Ed. Engl. 37 (1998) 2047-2067.

9. Yu, M. X.; Duan, J. P.; Lin, C. H.; Cheng, C. H.; Tao, Y. T.: Diaminoanthracene derivatives as high-performance green host electroluminescent materials. Chem. Mater. 14 (2002) 3958-3963.

10. Sheldrick, G. M.: SHELX-97. Programs for Crystal Structure Analysis. University of Göttingen, Germany 1997.

11. Farrugia, L. J.: ORTEP-3 for Windows - a version of ORTEP-III with a Graphical User Interface (GUI). J. Appl. Crystallogr. 30 (1997) 565-565.

12. Farrugia, L. J.: WinGX suite for small-molecule single-crystal crystallography. J. Appl. Crystallogr. 32 (1999) 837-838. 